\title{
Surgical treatment of congenital pseudoarthrosis of the clavicle: Our 22-year, single-center experience
}

\author{
Carmen Martínez-Aznar, $\mathrm{MD}^{1}{ }^{1}$, Isabel Parada-Avendaño, $\mathrm{MD}^{1}{ }^{(1 D}$, Victoria E Gómez-Palacio, MD, $\mathrm{PhD}^{1}$ (D), \\ Sandra Abando-Ruiz, MD ${ }^{1}$, Jorge Gil-Albarova, MD, $\mathrm{PhD}^{1,2}{ }^{(1)}$
}

${ }^{1}$ Orthopedic and Trauma Surgery Service, Miguel Servet University Hospital, Zaragoza, Spain

${ }^{2}$ Department of Surgery, Faculty of Medicine, University of Zaragoza, Zaragoza, Spain.

Congenital pseudarthrosis of the clavicle (CPC) is a rare entity. It was first reported by Fitzwilliams ${ }^{[1]}$ in 1910 and it is difficult to know the true incidence with around 200 cases published in the literature. The exact etiology is still unknown. The most accepted theory states that it is a failure of fusion of the two diaphyseal membranous ossification centers in the sixth intrauterine week of gestation ${ }^{[2]}$ and secondary to anatomic abnormalities which affect the subclavian artery, as in the presence of a prominent first rib. ${ }^{[3,4]} \mathrm{It}$ explains that most patients present right-sided CPC, and cases of left-sided are associated to dextrocardia. Bilateral cases are exceptional. ${ }^{[5]}$ It seems to be more common in females. Congenital pseudarthrosis of the clavicle can be diagnosed at birth or later in childhood, as a swelling in the middle-third clavicle but which is normally painless (Figure 1), being necessary to establish a differential diagnosis with

Received: November 03, 2020

Accepted: December 02, 2020

Published online: January 06, 2021

Correspondence: Carmen Martínez-Aznar, MD. Orthopedic and Trauma Surgery Service, Miguel Servet University Hospital, Paseo de Isabel la Católica, 50009 Zaragoza. Spain.

E-mail: cmartinezaznar@gmail.com

Doi: $10.5606 /$ ehc. 2021.79576

Citation: Martínez-Aznar C, Parada-Avendaño I, GómezPalacio VE, Abando-Ruiz S, Gil-Albarova J. Surgical treatment of congenital pseudoarthrosis of the clavicle: Our 22-year, singlecenter experience. Jt Dis Relat Surg 2021;32(1):224-229.

\section{(C2021 All right reserved by the Turkish Joint Diseases Foundation}

This is an open access article under the terms of the Creative Commons Attribution-NonCommercial License, which permits use, distribution and reproduction in any medium, provided the original work is properly cited and is not used for commercial purposes (http://creativecommons.org/licenses/by-nc/4.0/).

\section{ABSTRACT}

Objectives: This study reports the surgical outcomes in a patient cohort with congenital pseudarthrosis of the clavicle (CPC).

Patients and methods: A total of nine pediatric patients (5 males, 4 females; mean age: 4.43 years; range, 2 to 12 years) who were diagnosed with CPC and treated surgically with a minimum one-year follow-up between January 1996 and December 2018 were retrospectively analyzed. The patients were divided into two groups according to the fixation method as the Kirschner wire (K-wire; Group A, n=2) or stabilization with a plate (Group B, $n=7$ ). The physical function and symptoms were evaluated using the Quick Disabilities of the Arm, Shoulder and Hand (QuickDASH) scale.

Results: The mean age at the time of diagnosis was 2.95 (range, 0 to 12 ) years. Six cases were atrophic pseudarthrosis and three cases were hypertrophic. Radiographic consolidation occurred in all cases with a mean duration of $103.8 \pm 39.1$ days, indicating no significant difference between the groups ( $\mathrm{p}>0.05$ ). Complications registered were a K-wire breakage in a patient in Group A and an internal fixation plate loosening in a patient in Group B. The mean postoperative follow-up was $2.98 \pm 1.82$ years. The QuickDASH score was 0 points in all patients.

Conclusion: The early surgical indication based on refreshment of the pseudarthrosis focus, with bone autograft interposition when a failure exists to restore adequate clavicular length, and fixation are reliable alternatives with favorable clinical and radiological results in the mid- and long-term with fewer complications.

Keywords: Clavicle, congenital pseudarthrosis of clavicle, quick disabilities of the arm, shoulder and hand, surgical procedures.

obstetric fractures, post-traumatic nonunion, neurofibromatosis or cleidocranial dysostosis. ${ }^{[6,7]}$ It is possible that symptoms appear due to growth or increased activity. ${ }^{[8]}$ In rare cases, thoracic outlet syndrome or brachial plexus nerve compression are described ${ }^{[9]}$ There is no clear consensus on the optimal treatment for this entity, at what age or what surgical technique should be performed. Some authors have advocated for a more conservative 
treatment, contending that the difficulty to achieve a bony union and the fact that patients do not achieve better functional results with surgery. ${ }^{[10,11]}$ However, a surgical intervention is now widely accepted. Some authors who argue in favor of surgery conclude good functional outcomes in patients with evident deformity and/or related symptomatology. ${ }^{[12]}$

In the present study, we aimed to evaluate the surgical outcomes in a patient cohort with CPC treated in our center.

\section{PATIENTS AND METHODS}

This single-center, retrospective study was conducted at Orthopedic and Trauma Surgery Service, Miguel Servet University Hospital, Zaragoza, Spain between January 1996 and December 2018. All children diagnosed with CPC and treated surgically using one of two fixation techniques, namely Kirschner wire (K-wire) or plate fixation, ${ }^{[13]}$ and followed for minimum one year after surgery were included. Those having associated other pathologies (i.e., obstetric or traumatic fractures of the clavicle, neuromuscular disorders or cleidocranial dysplasia) were excluded. A total of 13 patients were diagnosed with CPC in our center; however, only nine of them ( 5 males, 4 females; mean age: 4.43 years; range, 2 to 12 years) fulfilled the inclusion criteria. Two patients did not undergo surgery due to associated cerebral palsy and major neurosensory disability. The other two patients were admitted to our center at the age of three months and eight months, respectively. The data were extracted from an institutional database and a written informed consent was obtained from each parent and/or legal guardian for the surgery. The study was conducted in accordance with the principles of the Declaration of Helsinki.

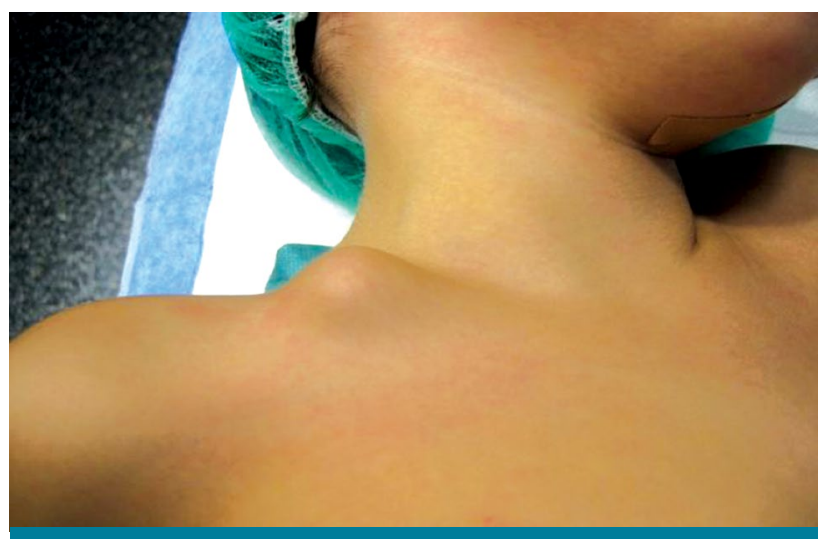

FIGURE 1. Clinical aspect of congenital pseudarthrosis of the clavicle.
Two methods of fixation during surgery were performed: The K-wire (Group $\mathrm{A}, \mathrm{n}=2$ ) or fixation with a plate (Group B, n=7). Fixation was performed with a K-wire in the two oldest cases, while the most common technique was a plate with 4 or 6 cortices on each side of the focus (Figure 2). Data were collected from medical records and radiological tests at the time of diagnosis and at six weeks, at three months,
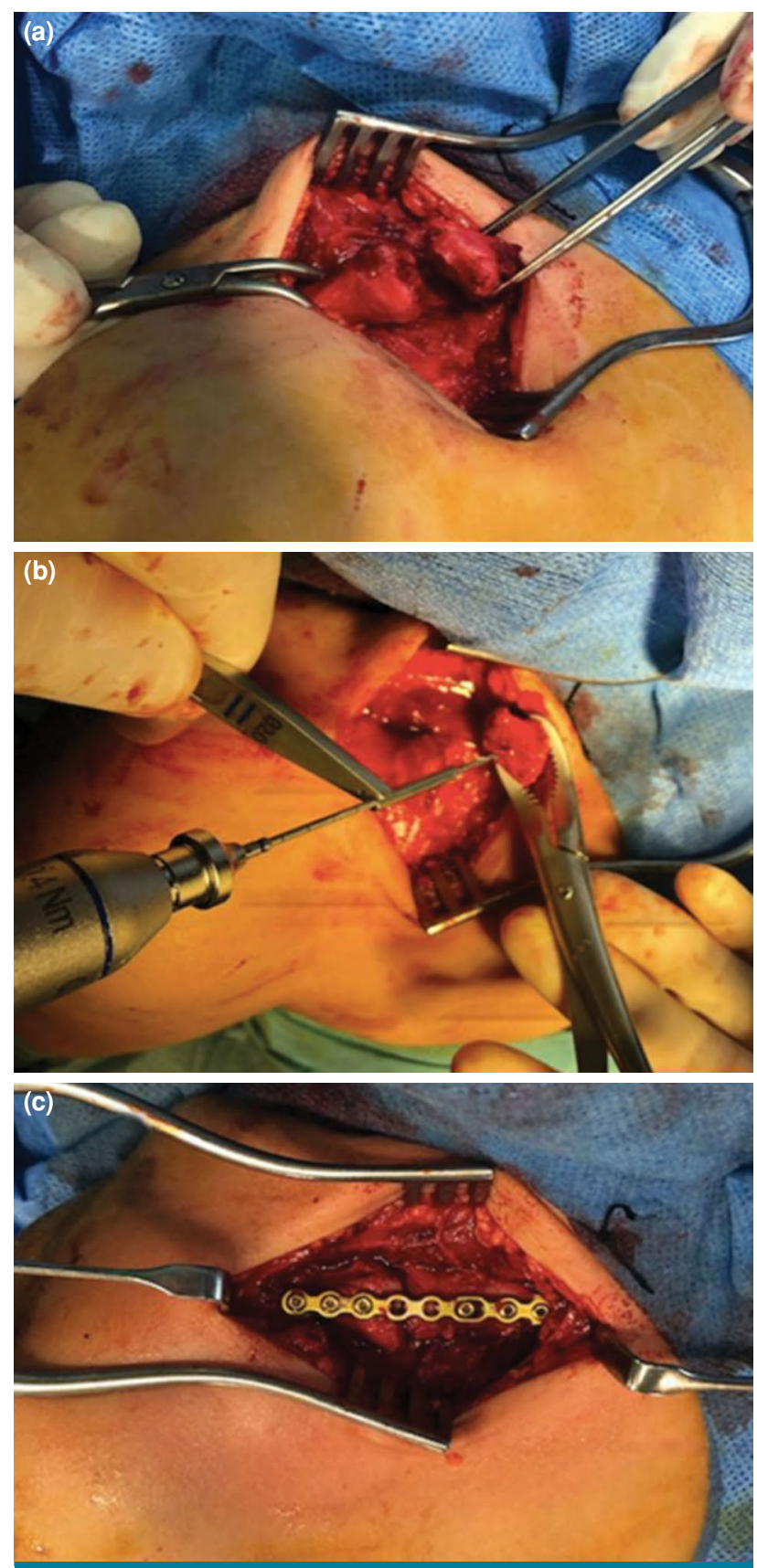

FIGURE 2. (a) An intraoperative image of congenital pseudarthrosis of the clavicle. (b) Focus of pseudarthrosis after refreshment and intramedullary drilling. (c) Fixation with a reconstruction plate. 
and at one year after surgery and after removal of the osteosynthesis material. Demographic and clinical characteristics of the patients such as sex, age at the time of diagnosis, affected side, symptoms, type of pseudarthrosis, age at the time of intervention, type of surgical treatment, radiographic bone healing time, results, complications, implant removal time, and follow-up were recorded. In order to measure the results of physical function and symptoms, we used the Quick Disabilities of the Arm, Shoulder and Hand (QuickDASH) scale during the postoperative visit, two or three months after removal of the material. ${ }^{[14]}$

The policy of our hospital at the time of this study was to offer surgical management to all patients with CPC who met inclusion criteria. The minimum age of the children at the time of operation was two years old and the reasons were cosmetic in all of them and/or discomfort with the backpack in the clavicular area in older patients. No patient presented pain at the time of diagnosis. All patients presented a swelling in the clavicular region at or soon after birth. Radiographically, we classified them as atrophic or hypertrophic pseudarthrosis. The technique consisted of a longitudinal incision over the superior surface of the clavicle, followed by refreshment the focus of pseudarthrosis and drilling the intramedullary canal in all cases. An autograft was used only in the atrophic patients at the bony edges and/or a gap after excision of the non-union. Postoperative care was similar in all patients; a sling was kept for an average of four weeks, allowing active shoulder mobilization afterwards, without requesting physiotherapy. Sports activity was not allowed for three months. All patients underwent a scheduled extraction of the osteosynthesis material.

\section{Statistical analysis}

Statistical analysis was performed using the IBM SPSS version 19.0 software (IBM Corp., Armonk, NY, USA). Continuous variables were expressed in mean \pm standard deviation (SD), median (min-max), while categorical variables were expressed in number and frequency. The Kolmogorov-Smirnov test, with Lilliefors correction, was performed to assess the normality distribution. The Fisher's exact test and the non-parametric Mann-Whitney $U$ test were used to compare the groups for non-normally distributed variables. A $p$ value of $<0.05$ was considered statistically significant.

\section{RESULTS}

Baseline demographic and clinical characteristics of the patients are shown in Table I.

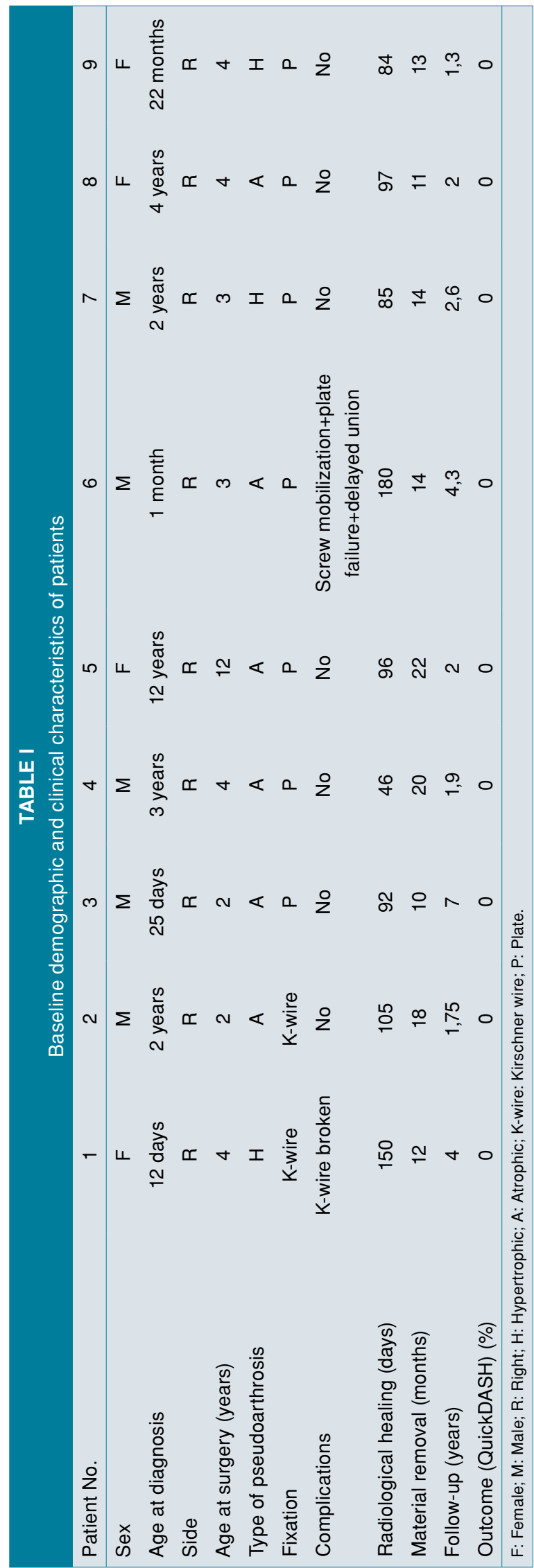



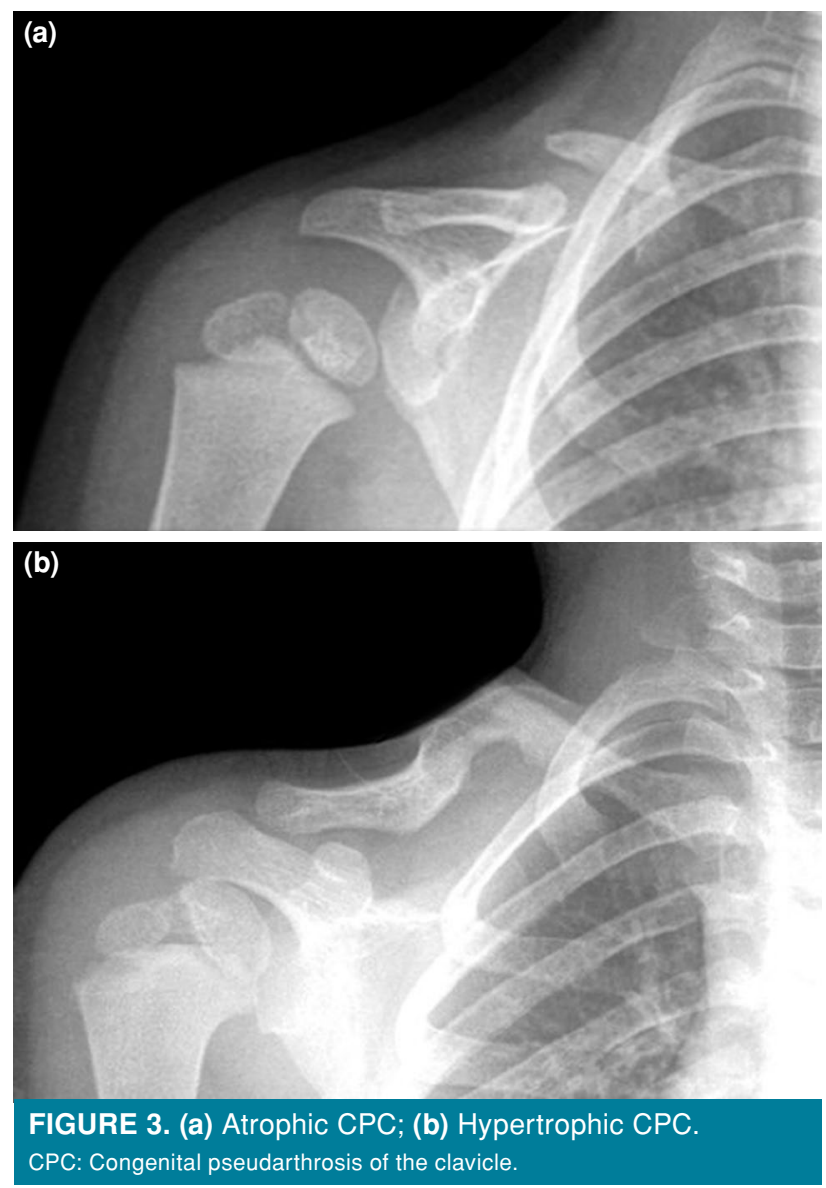

In all patients, the right side was affected. The mean age at the time of diagnosis was 2.95 (range, 0 to 12) years. On physical examination, all patients had a visible deformity over the midclavicular line and were neurovascularly intact. Hypertrophic pseudarthrosis was described in seven patients $(53.84 \%)$, three of whom were operated. The remaining six children $(46.15 \%)$ had atrophic characteristics and all were operated (Figure 3). In all of them, we used local autograft and allograft. Consolidation occurred in all patients with no significant differences regarding the time to achieve it $(\mathrm{p}>0.05)$.

In Group A, one patient presented a complication due to needle breakage one month after the intervention, which required early reoperation (Figure 4). In Group B, there were seven cases, one of which, with atrophic CPC, suffered from mobilization of the screws that had an impact on consolidation. This case underwent reintervention within two months through an open reduction, focus refreshment, and a new plate fixation with the additional corticocancellous iliac crest bone grafting. The result was satisfactory with a subsequent union. No donor site morbidity was observed.

Two patients presenting with complications with the material had a radiographic delay in
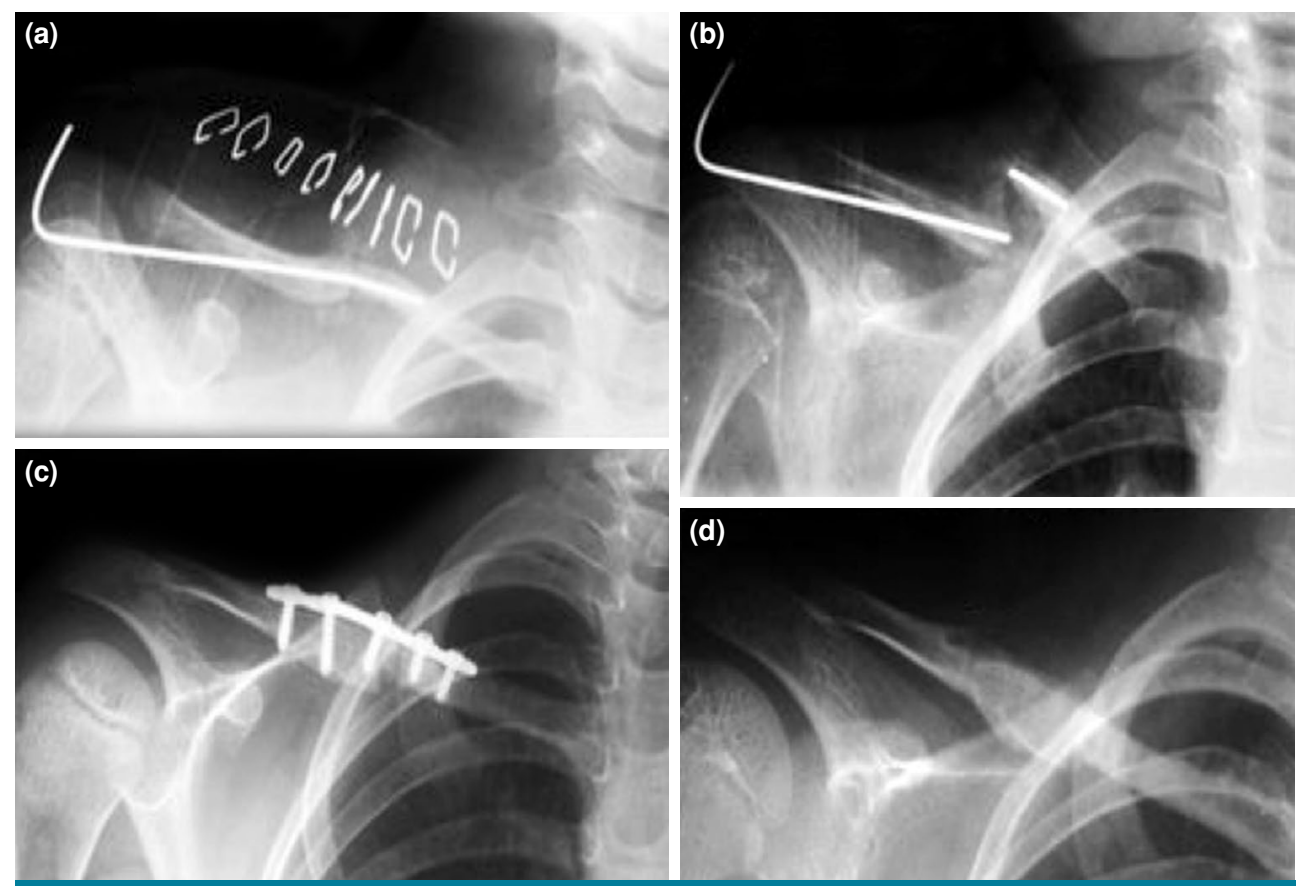

FIGURE 4. (a) Fixation with Kirschner wire; (b) Kirschner wire break; (c) Reintervention with focus refreshment and fixation with a plate adding corticocancellous iliac crest autograft; (d) One-year follow-up results. 
consolidation, reaching a mean of $165 \pm 21.2$ (range, 150 to 180) days. Good radiological consolidation was achieved in the rest of patients on $86.4 \pm 19.2$ (range, 46 to 105 ) days with no statistically significant differences between the groups.

Regarding sex, there were no significant differences in consolidation $(\mathrm{p}=0.858)$, as well as in the type of pseudarthrosis $(p=0.43)$. The mean postoperative follow-up was $2.98 \pm 1.82$ years.

The QuickDASH score was 0 points in all patients. Taking into account the first eight items about the ability to do activities, all patients presented complete shoulder mobility and ability to do them. None of the patients had pain.

The removal of the material in Group A occurred at a mean duration of four (range, 3 to 5) weeks, while the patients returned to the operating room at a mean duration of 14.88 (range, 10 to 22) months in Group B, as long as bone healing was observed on X-ray. The functional and quality of life results during follow-up were also satisfactory.

\section{DISCUSSION}

Congenital pseudarthrosis of the clavicle has a very low incidence. In the literature, there are few studies with long-term series ${ }^{[12,15,16]}$ and the article by Kim et al. $^{[15]}$ is one of them with the longest follow-up in 47 cases.

Currently, there is no consensus on the most optimal method and timing of treatment. Some authors have suggested a conservative treatment, if there are no symptoms, ${ }^{[10,11]}$ while others recommend surgery, regardless of the absence of symptoms. ${ }^{[17,18]}$ Surgical treatment can be established for cosmetic appearance, drooping of the shoulder, discomfort in the area, pain or functional symptoms ${ }^{[7]}$ or to avoid thoracic outlet syndrome or other vascular pathologies. ${ }^{[19]}$ In general, in all studies, the patient-reported surgery outcomes are favorable.

The mean age of intervention may be delayed in some cases due to the parent's decision who are unwilling for an early surgical treatment $(<6$ years of age) for their asymptomatic children; ${ }^{[18]}$ however, overall, many authors recommend performing the operation at the age of three to six years. ${ }^{[4,7,8,15,20,21]}$ In our study, the mean age was 4.43 years, consistent with previous findings. ${ }^{[2-12]}$ One of the reasons for this is the low rate of nonunion in this group of age, compared to children 18 months or older. ${ }^{[15]}$

There are different fixation methods used in the treatment including preservation of the periostal sleeve and fixation with non-absorbable suture alone in children under 18 months of age, ${ }^{[15]}$ screws alone or fully threaded pins. ${ }^{[22]}$ However, plates (reconstruction, compression or anatomical) and $\mathrm{K}$-wires are the most frequently used methods.

Although there are several reports advocating K-wire fixation, ${ }^{[8,12,17]}$ many of them were written before the most common use of anatomical and locking plates. Complications with K-wires fixation include pin-tract infections, breakages, delayed unions ${ }^{[12]}$ and migration taking into account the proximity of subclavian artery and brachial plexus. ${ }^{[22]}$ There are no published cases in which the subclavian artery or brachial plexus have been injured with the use of plate fixation.

The use of bone graft is considered important in the treatment of CPC to increase the union rate. ${ }^{[16,21]}$ The use of allogenic graft has been described, ${ }^{[12,18]}$ and a combination of local autograft and allograft has been reported, as well. ${ }^{[2]}$ However, it is preferred autologous iliac crest bone thanks to its greater biological benefits, ${ }^{[1,16]}$ taking into account a remained and prolonged pain in the donor area. To solve this complication, Abdellaoui et al. ${ }^{[23]}$ proposed a modified Masquelet technique, as an alternative, to obtain complete union of the focus without graft interposition and with minimal aesthetic defect.

In our study, the radiological evidence of consolidation was obtained at a mean duration of $103.8 \pm 39.1$ days, consistent with the studies of Chandran et al. ${ }^{[20]}$ and Ettl et al. ${ }^{[24]}$

Salvage procedures after surgical treatment complications consist in most of the cases of revision surgery, repeating resection of the pseudarthrosis, and placement of bone graft and internal fixation; however, there are some reports using vascularized bone grafting ${ }^{[25]}$ or vascularized medial femoral condyle flap, ${ }^{[26]}$ although they can never be considered the first choice in the management of CPC.

The main limitation of our study lies in its retrospective design with Level of Evidence IV, without a control group of conservative treatment. In addition, the small sample size did not allow for many statistical comparisons of subgroup analyses. Despite these limitations, the current study details treatment options for these patients and improves our understanding of the results of surgical treatment. Our case series is also comparable with previous published studies.

As a result of our experience and the literature review, we suggest that there is no clinical difference between earlier versus late operation; however, we advise an early surgical treatment performing a 
refreshment of the pseudarthrosis focus, using always autologous bone graft in atrophic CPC or when a gap appears between the fragments, and stable fixation. Between four to six years of age, it seems to be reasonable to achieve a good fixation and adaptation of a plate according to the size of the clavicle. It can also avoid difficulties which may occur in older children with a larger size of the gap and a possible longer time to heal. This approach may prevent the problems associated with an abnormal anatomy of the clavicle. Regarding fixation, based on our small cohort, it is difficult to reach a firm conclusion; however, our practice has changed from the K-wires to plate fixation as the first choice of treatment.

In conclusion, the scheduled early surgical indication ( $<6$ years of age) based on refreshment of the pseudarthrosis focus, with bone autograft interposition when a gap exists, and fixation are reliable alternatives with favorable clinical and radiological results in the mid- and long-term. It also yields minimal complications and improves aesthetics, avoiding possible future problems. We recommend surgical treatment as of four years of age using plate fixation as the first option.

\section{Declaration of conflicting interests}

The authors declared no conflicts of interest with respect to the authorship and/or publication of this article.

\section{Funding}

The authors received no financial support for the research and/or authorship of this article.

\section{REFERENCES}

1. Fitzwilliams DCL. Hereditary cranio-cleido-dysostosis. Lancet 1910;2:1466-75.

2. Fawcett. The Development and Ossification of the Human Clavicle. J Anat Physiol 1913;47:225-34.

3. Lloyd-Roberts GC, Apley AG, Owen R. Reflections upon the aetiology of congenital pseudarthrosis of the clavicle. With a note on cranio-cleido dysostosis. J Bone Joint Surg [Br] 1975;57:24-9.

4. Gomez-Brouchet A, Sales de Gauzy J, Accadbled F, Abid A, Delisle MB, Cahuzac JP. Congenital pseudarthrosis of the clavicle: a histopathological study in five patients. J Pediatr Orthop B 2004;13:399-401.

5. Nieto Gil A, Gómez Navalón A, Zorrilla Ribot P. Bilateral congenital seudarthrosis of the clavicle. A clinical case. Rev Esp Cir Ortop Traumatol 2016;60:397-9.

6. Chalfant JS, Sanchez TR. Congenital Clavicular Pseudoarthosis-How to Differentiate It From the More Common Clavicular Fractures. Pediatr Emerg Care 2019;35:e37-e39.

7. Persiani P, Molayem I, Villani C, Cadilhac C, Glorion C. Surgical treatment of congenital pseudarthrosis of the clavicle: a report on 17 cases. Acta Orthop Belg 2008;74:161-6.
8. Gibson DA, Carroll N. Congenital pseudarthrosis of the clavicle. J Bone Joint Surg [Br] 1970;52:629-43.

9. Watson HI, Hopper GP, Kovacs P. Congenital pseudarthrosis of the clavicle causing thoracic outlet syndrome. BMJ Case Rep 2013;2013:bcr2013010437.

10. O'Leary E, Elsayed S, Mukherjee A, Tayton K. Familial pseudarthrosis of the clavicle: does it need treatment? Acta Orthop Belg 2008;74:437-40.

11. Shalom A, Khermosh $\mathrm{O}$, Wientroub S. The natural history of congenital pseudarthrosis of the clavicle. J Bone Joint Surg [Br] 1994;76:846-7.

12. Di Gennaro GL, Cravino M, Martinelli A, Berardi E, Rao A, Stilli S, et al. Congenital pseudarthrosis of the clavicle: a report on 27 cases. J Shoulder Elbow Surg 2017;26:e65-e70.

13. Atik OS. Is there something new and interesting in my article? Eklem Hastalik Cerrahisi 2019;30:69.

14. Kennedy CA, Beaton DE, Smith P, Van Eerd D, Tang K, Inrig T, et al. Measurement properties of the QuickDASH (disabilities of the arm, shoulder and hand) outcome measure and cross-cultural adaptations of the QuickDASH: a systematic review. Qual Life Res 2013;22:2509-47.

15. Kim AE, Vuillermin CB, Bae DS, Samora JB, Waters PM, Bauer AS. Congenital pseudarthrosis of the clavicle: surgical decision making and outcomes. J Shoulder Elbow Surg 2020;29:302-7.

16. Owen R. Congenital pseudarthrosis of the clavicle. J Bone Joint Surg [Br] 1970;52:644-52.

17. Lorente Molto FJ, Bonete Lluch DJ, Garrido IM. Congenital pseudarthrosis of the clavicle: a proposal for early surgical treatment. J Pediatr Orthop 2001;21:689-93.

18. Studer K, Baker MP, Krieg AH. Operative treatment of congenital pseudarthrosis of the clavicle: a single-centre experience. J Pediatr Orthop B 2017;26:245-49.

19. Currarino G, Herring JA. Congenital pseudarthrosis of the clavicle. Pediatr Radiol 2009;39:1343-9.

20. Chandran P, George H, James LA. Congenital clavicular pseudarthrosis: comparison of two treatment methods. J Child Orthop 2011;5:1-4.

21. Cadilhac C, Fenoll B, Peretti A, Padovani JP, Pouliquen JC, Rigault P. Congenital pseudarthrosis of the clavicle: 25 childhood cases. Rev Chir Orthop Reparatrice Appar Mot 2000;86:575-80.

22. Toledo LC, MacEwen GD. Severe complication of surgical treatment of congenital pseudarthrosis of the clavicle. Clin Orthop Relat Res 1979;(139):64-7.

23. Abdellaoui H, Atarraf K, Chater L, Afifi MA. Congenital pseudarthrosis of the clavicle treated by Masquelet technique. BMJ Case Rep 2017;2017:bcr2017221557.

24. Ettl V, Wild A, Krauspe R, Raab P. Surgical treatment of congenital pseudarthrosis of the clavicle: a report of three cases and review of the literature. Eur J Pediatr Surg 2005;15:56-60.

25. Glotzbecker MP, Shin EK, Chen NC, Labow BI, Waters PM. Salvage reconstruction of congenital pseudarthrosis of the clavicle with vascularized fibular graft after failed operative treatment: a case report. J Pediatr Orthop 2009;29:411-5.

26. Murai A, Tada K, Nakada M, Matsuta M, Hayashi K, Tsuchiya $H$. Use of a free vascularized medial femoral condyle flap for revision surgery in a pediatric patient with congenital pseudarthrosis of the clavicle. Case Rep Orthop 2020;2020:8872934. 